\title{
Una Política Extractiva Petrolera en un Estado de Derechos y Justicia: Una Visión Crítica de la Política Pública en el Ecuador
}

\author{
Oil Extraction Policy under the Rule of Law and Justice: A \\ Critical View of Public Policies in Ecuador
}

\author{
Dr. Felipe Castro León \\ Investigador Jurídico, Dirección Jurídica CES \\ Artículo Original (Investigación) \\ RFJ, No. 1, 2017, pp. 111-136, ISSN 2588-0837
}

RESUMEN: A lo largo de este trabajo se busca analizar críticamente al extractivismo petrolero dentro del marco de acción del Estado, en específico como política pública. Se desarrolla el marco constitucional y legal que permite la realización de este tipo de actividades, y la institucionalidad que la administra y gestiona estas actividades. Posteriormente, se analiza las características del extractivismo respecto a la territorialización de los proyectos, la vinculación de las rentas extractivas al desarrollo económico del país, y los impactos ambientales que producen las actividades extractivas. Con este marco de análisis, se pudo observar cómo la política extractiva petrolera en el Ecuador ha sido a lo largo de los años una política de expansión de las fronteras de extracción de petróleo. Finalmente, dentro de un Estado de derechos y justicia que privilegia la realización de los derechos de las personas y la naturaleza, se pudo visibilizar la necesidad de un replanteamiento de esta política pública frente a un balance no muy positivo a la hora de su implementación.

PALABRAS CLAVE: Extractivismo, política pública, política extractiva petrolera, capacidad productiva, reservas petroleras.

ABSTRACT: This work is based on a critical analysis of oil exploitation as public policy: firstly, the Constitutional and legal regulations that allow for oil exploitation activities to be carried out and the institutions that administrate these activities are examined; secondly, the characteristics of oil exploitation in regards to territorial appropriation, the linking of oil incomes with economic development, and the 
environmental impacts are analyzed. Under this framework, the expansion of oil extraction borders as part of Ecuadorian extractive oil policy is examined.. Lastly, in a rule of law and justice State that favors human and environmental rights, a need to rethink this public policy is seen, given the less than positive balance upon its implementation.

KEY WORDS: Extractivism, public policy, oil exploitation public policy, productive capacity, oil reserves.

\section{INTRODUCCIÓN}

La economía del Ecuador se ha caracterizado por ser primario-exportadora, y el Estado se ha valido del extractivismo para justificar una vía a lo que comúnmente se conoce como desarrollo. Este extractivismo se ha justificado como el modelo necesario para garantiza los derechos de la sociedad ecuatoriana a lo largo de los años, pero los años de explotación de recursos naturales ha demostrado lo contrario.

Pese a esto, el extractivismo no es únicamente la extracción de recursos naturales, sino que representa un fenómeno social que tiene consecuencias en el ejercicio de las poblaciones aledañas a los proyectos puesto que utiliza la violencia como medio de despojo de los territorios. Esto ha ocurrido sin duda con la explotación del petróleo, la cual ha afectado a las poblaciones dentro de la zona de influencia de los proyectos extractivos ya que ha causado daños ambientales y culturales, junto con conflictividad social, y acompañado de políticas para la colonización de territorios.

En este punto es importante señalar el papel que ha tenido el Derecho, al ser esta la herramienta que ha justificado, y, hasta cierto punto, legitimado la realización de este tipo de actividades. Un aspecto a considerar en este contexto se refiere a la posibilidad que otorga tanto la Constitución como las normas secundarias a la realización de actividades extractivas, pero que deben enmarcarse también en el amplio desarrollo de los derechos reconocidos en ella.

Si bien este no representa un debate nuevo, sin embargo, se encuentra aun plenamente vigente. La necesidad de analizar la forma en cómo estas actividades han sido implantadas por el Estado resulta imperativo en un escenario donde las alternativas sociales al extractivismo todavía no son difundidas en la sociedad de forma efectiva. 
Por estos motivos, se busca a través del presente trabajo analizar el papel del Estado y el Derecho de forma crítica frente a la ejecución de actividades que tienen influencia principalmente en los derechos de las personas y las comunidades.

\section{CONFIGURACIÓN DEL EXTRACTIVISMO PETROLERO COMO POLÍTICA PÚBLICA}

Para entender cómo se configura como una política pública es necesario repasar algunas definiciones establecidas desde la teoría. Tenemos que política pública es todo lo que los gobiernos deciden hacer o no hacer (Dye, 2012). En esta línea, son entendidas como las decisiones políticas, tomadas entre varias opciones, desde y para el marco institucional del Estado, que involucran a las personas, y que buscan obtener ciertos resultados (Kelman, 1992, 23).

Sin embargo, hay que tomar en cuenta que casi nunca una política pública es una sola decisión, ya que realmente involucra un conjunto de decisiones simultáneas o secuenciales (Eslava y Puente, 26). Estas decisiones transformadas en políticas públicas, según el mismo Kelman, son producto de una elección que constituyen los rasgos fundamentales del sistema político y de los requerimientos de la sociedad (Kelman, 1992, 24). Dicho en otras palabras, la decisión o decisiones tomadas en virtud de un requerimiento social, y dentro del ámbito jurídico-institucional del Estado, se denomina política pública.

A nivel gubernamental ecuatoriano, la SENPLADES estableció la política pública como un curso de acción de la gestión pública que institucionaliza la intervención pública en respuesta a un problema social identificado como prioritario, y que se convierte de esta manera en materia de política de Estado (SENPLADES, 2011, 10).

En este contexto, la política extractiva petrolera se refiere a la inscripción del extractivismo en el curso de acción de la gestión pública; al ser el extractivismo definido como un tipo de extracción de recursos naturales en gran volumen o alta intensidad, en donde dichos recursos son exportados como materias primas sin procesar, o con un procesamiento mínimo (Gudynas, 2013, 3), en función de la demanda de los países centrales (Acosta, 2015). 
Los recursos naturales extraídos por excelencia son principalmente productos hidrocarburíferos y mineros, aunque se puede extender también al extractivismo agrario, forestal e inclusive pesquero (Acosta, 2015). En el caso del petróleo, las actividades extractivas se relacionan con la búsqueda de estratos subterráneos o reservorios con hidrocarburos denominados yacimientos, continúa con su extracción a la superficie, $y$ finalmente son transportado para su exportación y/o refinación (Játiva, 2010, 202-203).

En consecuencia, la política extractiva petrolera involucra las acciones del Estado para emprender o dar lugar a la realización de actividades de extracción de alta intensidad de recursos hidrocarburíferos.

\section{REGULACIÓN DE LA POLÍTICA EXTRACTIVA PETROLE- RA EN EL MARCO CONSTITUCIONAL DEL ECUADOR}

Una vez visto que el extractivismo petrolero se puede configurar como una política pública en el marco de acciones del Estado, es necesario abordar cómo la legislación avala la realización de este tipo de actividades y cómo estas se pueden realizar.

En esta línea, es importante destacar el papel que tiene el Estado, que, con su monopolio de la violencia y sus definiciones de legalidad, juega un rol crucial al respaldar y promover los procesos de apropiación de recursos naturales (Harvey, 2005, 133). Por esta razón, y con apoyo de la teoría económica, el derecho ha dado paso a la mercantilización de la naturaleza (lógica de compra y venta) por medio de la definición de los derechos de propiedad sobre los recursos naturales (Dávalos, 2013), lo que permite su explotación y su intercambio en el mercado mundial (Hernández, Rodríguez y Moreno, 2004, 42).

Actualmente, esta regulación sobre la propiedad de los recursos naturales se puede encontrar en la Constitución ecuatoriana, cuyos artículos: 1,317 , y 408, determinan que los recursos naturales no renovables ubicados en el territorio del Ecuador pertenecen al patrimonio inalienable, irrenunciable e imprescriptible del Estado.

Esta determinación de la propiedad le permite al Estado la administración, la regulación, el control y la gestión los recursos naturales no renovables de acuerdo al artículo 313 de la Constitución. Incluso goza 
de capacidad de expropiación de terrenos y otros bienes que fuesen necesarios para el desarrollo de las actividades extractivas (artículo 4 de la Ley de Hidrocarburos), lo que le permite constituir redes para la circulación de territorios y de los mismos recursos (carreteras, oleoductos, estaciones de acopio, entre otras).

De igual forma, estos recursos naturales son considerados gravitantes para la estructura económica del país, razón por la que se lo adopta en la categoría de sector extractivo, por su decisiva influencia económica, social, política o ambiental, según el artículo 313 de la Constitución. Esto concuerda con la visión que le da el Plan Nacional de Desarrollo a los recursos petroleros como fuente de riqueza para el país, y con incidencia que tienen en todo el país puesto que los ingresos que genera comprometen a todo el Ecuador (Corte Constitucional del Ecuador, Caso 001-09-SEE-CC, 2009).

La consecuencia de la importancia que se le ha dado a la extracción de recursos naturales en la Constitución le permite al Estado adoptar una presencia más activa en las actividades vinculadas a este sector. Este rol activo se encuentra principalmente en la gestión de estos sectores, ya que el artículo 315 de la Constitución da lugar a que la explotación de recursos naturales se lo realice directamente por parte de empresas públicas y empresas mixtas con mayoría accionaria del Estado, mientras que en el artículo siguiente deja de forma excepcional la participación de los privados.

\section{REGULACIÓN DEL MARCO INSTITUCIONAL RESPECTO AL EXTRACTIVISMO PETROLERO}

Ahora es necesario establecer el marco de actores institucionales que pone en marcha las actividades que comprende la política extractiva petrolera, y que, debido a la activa participación del Estado, pertenecen al sector público. Por estas razones, a partir de la Constitución y la Ley, se desarrollan las potestades para la realización de varios tipos de actividades en varios niveles.

El primer nivel se refiere a las entidades de planificación y definición de la política pública. De acuerdo al artículo 141 de la Constitución, la Función Ejecutiva tiene la competencia de rectoría, planificación, ejecución y evaluación de las políticas públicas nacionales y planes que se creen para ejecutarlas. En este punto, el instrumento bajo el cual 
se hace efectiva y se evidencia la competencia de la Función Ejecutiva respecto a la política pública es el Plan Nacional de Desarrollo.

Así mismo, la Ley de Hidrocarburos confirma lo establecido en la Constitución, y va más allá al determinar la formulación de la política pública de hidrocarburos le corresponde al Ministerio de ramo y a la Secretaría de Hidrocarburos. El Ministerio de ramo establecido fue el de Hidrocarburos según el Decreto Ejecutivo 578, publicado en el Registro Oficial 448 de 28 de febrero de 2015.

En el segundo nivel están las instituciones que tienen la tarea de regulación y el control de los sectores estratégicos (artículo 315), de acuerdo a la Constitución y la ley. En un primer momento, la Asamblea Nacional es el órgano encargado de expedir, codificar, reformar y derogar las leyes, e interpretarlas con carácter generalmente obligatorio (artículo 120.6). Estas leyes pueden referirse a las actividades que comprenden los sectores estratégicos y por ende los recursos naturales, así como regular el ejercicio de los derechos y garantías, tipificar y establecer sanciones, y regular la organización y funcionamiento de las instituciones creadas por la Constitución y de los organismos de control y regulación (artículos 132 y 133).

Por otro lado, la Ley de Hidrocarburos, la norma con rango legal vigente, también establece instituciones con capacidad de regulación y control. Se determina que el mismo Ministerio de Hidrocarburos está facultado para dictar los reglamentos y las disposiciones (Corte Constitucional del Ecuador, Caso 0004-10-SIN-CC, 2010). Por su parte, la Agencia de Regulación y Control Hidrocarburífero (ARCH), como institución de derecho público adscrita al Ministerio, se encarga de "regular, controlar y fiscalizar las actividades técnicas y operacionales en las diferentes fases de la industria hidrocarburífera". Las fases a las que se hace mención están definidas en el artículo 9 de la Ley y se refieren a la prospección, exploración, explotación, refinación, industrialización, almacenamiento, transporte y comercialización de los hidrocarburos y de sus derivados.

Junto con el Ministerio y la ARCH, funciona la Secretaría de Hidrocarburos como entidad adscrita al Ministerio Sectorial. De acuerdo al artículo 6-A de la Ley de Hidrocarburos y los artículos 3, 5, 7, y 10 del Reglamento de Aplicación de la Ley Reformatoria a la Ley de Hidrocarburos autoriza las actividades de exploración y explotación de hidrocarburos en el caso de empresas públicas; celebra contratos de exploración y explotación de hidrocarburos con empresas mixtas, estatales de los 
países de la comunidad internacional; y lleva a cabo un proceso de licitación, adjudicación, y celebración de contrato con empresas privadas.

Además, se encuentra la Autoridad Ambiental Nacional (Ministerio del Ambiente), quien tiene la competencia de otorgar licencia ambiental (permiso ambiental), cuando se trata de una actividad de alto impacto y riesgo ambiental como la hidrocarburífera, debido, a que según, el artículo 9 del Libro VI sobre la Calidad Ambiental del Texto Unificado de Legislación Secundaria de Medio Ambiente es parte de los sectores estratégicos determinados por la Constitución. Según el artículo 26 del mismo cuerpo normativo, es competencia exclusiva el manejo por parte de la Autoridad Ambiental Nacional respecto a todos los proyectos, obras o actividades que intersequen con el Sistema Nacional de Áreas Protegidas (SNAP), Bosques y Vegetación Protectores (BVP), Patrimonio Forestal del Estado (PFE).

Finalmente, en un tercer nivel encontramos a las entidades encargadas de la gestión y administración de los recursos naturales. De acuerdo a la Corte Constitucional, la gestión y la administración son sinónimos, y tienen relación con las actividades de organización, operación, dirección, toma de decisiones que el Estado hace respecto de los sectores estratégicos (Corte Constitucional del Ecuador, Caso 001-12-SIC-CC, 2012).

Como ya se estableció, a este nivel le corresponde a las empresas públicas, mixtas, y de forma excepcional a la iniciativa privada y a la economía popular y solidaria. Actualmente, los encargados de la gestión de los recursos hidrocarburíferos son:

- Empresas públicas: 1) EP Petroamazonas, encargada del manejo del "upstream" hidrocarburífero -exploración y explotación de los campos de petróleo y gas- (SENPLADES, 2013, 45); 2) EP Petroecuador, encargada del "downstream" hidrocarburífero -transporte, refinación y comercialización de los combustibles-, además de las importaciones y exportaciones de los derivados del petróleo; EP Petroecuador (SENPLADES, 2013, 45); y 3) EP Flopec, encargada del transporte marítimo de hidrocarburos (SENPLADES, 2013, 45).

- Empresas mixtas: 1) Operaciones Río Napo CEM, encargada de la operación del campo Sacha, cuyo paquete accionario le pertenece en un $70 \%$ a Petroamazonas EP y el $30 \%$ a PDVSA (SENPLADES, 2013, 45); y 2) Refinería del Pacífico CEM, creada para la construcción y operación del complejo refinador en Manabí, cuyo paquete 
accionario actualmente le corresponde a EP Petroecuador en un $51 \%$, y a PDVSA en un $49 \%$ (SENPLADES, 2013, 45).

- Empresas privadas: el Estado tiene los Contratos de Prestación de Servicios con Pago de Tarifa con 14 operadoras privadas que trabajan actualmente en 17 campos y bloques (Ministerio de Hidrocarburos, 2015).

En suma, todas estas instituciones permiten la ejecución de una política extractiva petrolera por parte del Estado, es decir, dan paso a las actividades para la extracción de recursos hidrocarburíferos en el Ecuador por medio de diferentes niveles en el sector público e incluso en el privado.

\section{GESTIÓN DE LAS ACTIVIDADES EXTRACTIVAS PETRO- LERAS}

Con la reforma en el año 2010 a la Ley de Hidrocarburos se buscó mayor participación estatal en la gestión de estos recursos. Esta reforma buscó cumplir con el postulado constitucional de la gestión directa de los recursos naturales a través del Estado, y encontró en el repunte de la modalidad de contratación bajo la prestación de servicios el instrumento para realizarlo, sin dejar de mencionar la coyuntura de los altos precios del petróleo en el mercado mundial que motivó a promoverla.

La principal característica de este tipo de contrato es que le permite al Estado no ceder su propiedad sobre crudo producido, y solamente se limita a contratar a la Operadora para que explore y explote sus campos por un tiempo determinado (Molina, 2014, 6). En virtud de lo señalado, el Estado debe cancelar una tarifa fija por cada barril producido y se reserva un margen de soberanía con el que participa con un $25 \%$ del ingreso bruto generado a través de la explotación de cualquier área destinada para el efecto (Molina, 2014, 115).

En la práctica, la contratación bajo prestación de servicios asegura a las compañías un ingreso permanente, mientras que, para el país, sus ingresos están determinados por el comportamiento del precio internacional del hidrocarburo (Fundación Mil Hojas, 2015). 


\section{CARACTERIZACIÓN DEL EXTRACTIVISMO PETROLERO EN EL ECUADOR}

Desarrollada la regulación, la institucionalidad, y la gestión de la política extractiva petrolera, no se puede dejar atrás la forma en cómo estas actividades son realizadas y sus impactos en las comunidades y la naturaleza. En este sentido, las prácticas del extractivismo han demostrado que una condición necesaria para poder llevar a cabo estas actividades es la violencia y la vulneración a derechos humanos y de la naturaleza, lo cual ha sido calificado en un caso más agudo como extracción (Gudynas, 2013, 15). Para poder abordar estas características, se ha planteado clasificarlas de la siguiente forma: territorialización, daños ambientales, y vinculación con el desarrollo económico.

\subsection{Territorialización}

Una característica de los proyectos extractivos es su territorialización, es decir, la localización geográfica de las actividades de extracción de recursos naturales que conlleva tres consecuencias: gobernanza o capacidad de decisión sobre los territorios, apropiación material del territorio, y la constitución de redes y flujos para la circulación entre territorios (Lobos, 2013, 47).

En Ecuador, los proyectos extractivos petroleros se han localizado en el oriente del país, especialmente en la Amazonía centro norte. Este ha sido tradicionalmente el hogar de varias nacionalidades y pueblos indígenas, los cuales hasta la actualidad se enfrentan a un proceso de contacto y colonización, y de pérdida progresiva de sus territorios para facilitar las actividades petroleras. Este proceso fue apoyado por varios actores, entre ellos empresas petroleras y el Estado, quienes provocaron la territorialización de los proyectos extractivos al permitir la gobernanza, la apropiación, y la circulación sobre los territorios de sus legítimos dueños.

Respecto al Estado, encontramos que la determinación de la propiedad de estos recursos por medio de la legislación le permite adoptar decisiones respecto al territorio y apropiarse materialmente de éste cuando encuentre recursos susceptibles de apropiación como el petróleo. 


\subsection{Daños ambientales}

Otro aspecto que caracteriza al extractivismo petrolero es la gran intensidad con la que se realiza estas actividades, lo cual viene acompañado de daños ambientales catastróficos (Fundación Heinrich Böll, $2015,2)$. Incluso, en muchos casos, si se contabilizan los costos económicos de los impactos sociales, ambientales y productivos de la extracción del petróleo o de los minerales, desaparecen muchos de los beneficios económicos de estas actividades (Acosta, 2015, 13).

En Ecuador, existen un sinnúmero de casos que comprueban la forma en cómo el extractivismo petrolero se ha llevado a cabo. Cabe mencionar, por ejemplo, uno de los mayores desastres ambientales petroleros registrados en el país, y que corresponde a los impactos generados por Texaco, en donde se vertieron 18 mil millones de galones de desechos tóxicos en aguas de formación, con la operación de 339 pozos (Serrano Narváez, 2013, 23).

Por esta razón, el extractivismo no comprende la simple extracción de recursos naturales, sino que implica procesos de control territorial y localización de afectaciones ambientales dentro de dicho territorio. Esta situación produce a su vez judicialización social, valorización mercantil de recursos intangibles, derechos de propiedad sobre recursos territoriales en mercados altamente integrados sobre todo mercados financieros, dinámicas de política demográfica y de control poblacional a través de bonos de la pobreza, entre otras (Dávalos, 2013, 2).

\subsection{Vinculación con el desarrollo económico}

El extractivismo ha tenido una vinculación con el desarrollo económico de los países, pero que ha expresado una doble vulnerabilidad.

En primer lugar, la vulnerabilidad de los Estados se expresa cuando existe un elevado precio del recurso natural en el mercado, o el descubrimiento de una nueva fuente o yacimiento desata un boom de exportación primaria se puede recaer en la denominada enfermedad holandesa (Acosta, 2009, 42-43). Esto comprende un proceso por medio del cual la economía se distorsiona por un masivo ingreso de divisas, lo que provoca una sobrevaluación de la moneda y la consecuente pérdida de competitividad del sector manufacturero, junto con una 
migración de los recursos casi exclusivamente al sector primario en auge (Acosta, 2009, 153).

Al respecto, hay que tomar en cuenta que la economía ecuatoriana ha sido tradicionalmente primaria-exportadora (Acosta, 2006, 116), razón por la cual en el período 2007-2013, por ejemplo, el crecimiento económico del país tuvo relación directa con el precio mundial de las materias primas (Dávalos, 2014, 123). En este escenario, se ha asociado al extractivismo como una batería de políticas sociales dirigidas a los sectores más vulnerables (Svampa, 2013, 37-38), tal como lo señala el Plan Nacional del Buen Vivir en los períodos 20092013 y 2013-2017.

Pese a aquello, en el período 2007-2013 se ha comprobado que la renta extractiva en el Ecuador nunca financió de manera importante al sector social, sino produjo distorsiones en la economía del país al crear una burbuja de consumo (Dávalos, 2014, 143), y no ha cumplido los objetivos del cambio de una matriz productiva, puesto que ha existido un proceso de desindustrialización (Dávalos, 2014, 121). Más bien, se trata de un extractivismo agresivo, que utiliza el discurso del aprovechamiento de materias primas por las altas tasas de ganancia y la dependencia de sus exportaciones para lograr las metas de crecimiento económico (Azamar y Ponce, 2014, 145-146) como un mecanismo para justificar (Gudynas, 2009, 209) y generar un consenso social sobre el extractivismo como un mal necesario para el desarrollo (Dávalos, 2014, 184).

Por otro lado, la vulnerabilidad de los Estados también se expresa frente a fuertes caídas de los precios (Fundación Heinrich Böll, 2015, 3 ), lo que conlleva a un desbalance en la balanza de pagos al verse disminuidos los ingresos económicos (Acosta, 2009, 154), y provoca interrupciones inesperadas y quizás irreversibles en proyectos a largo plazo que requieren años de financiación pública y gestión sostenida (Oxfam Internacional, 2009, 24). Esto se agrava aún más cuando existe una concentración de la exportación en unos pocos productos primarios como parte de la estructura productiva de la economía primaria (Petit Primera, 2013, 128-129).

Esta situación también sucede en Ecuador, donde alrededor del 11\% de los ingresos del Estado provienen de las ventas de crudo, razón por la que la volatilidad de su precio tiene consecuencias directas en las decisiones de política pública, las que se ven comprometidas cuando 
su precio disminuye (Facultad de Economía de la PUCE, 2015, 30). Por esta razón, en el año 2015, frente a la caída del precio internacional del petróleo, se realizó un recorte en el Presupuesto General del Estado de alrededor 2200 millones de dólares (El Universo, 2015).

Además, también hay que tomar en cuenta que el modelo de contratación implantado en la reforma a la Ley de Hidrocarburos no ha sido rentable en este escenario. Esto se debe a que las empresas petroleras, por prestación de servicios, han venido cobrándole al Estado, en promedio, USD 29,69 por cada barril extraído, cuando esos mismos barriles se comercializan en el mercado internacional en un promedio de USD 35 en el 2015 (Redacción Plan V, 2015). Esta situación se compara con el promedio del precio del barril de petróleo de USD 70 entre el 2007 y el 2014 (Fundación Mil Hojas, 2015), cuando esta modalidad representaba mayores ingresos para el país.

En suma, el extractivismo petrolero es un proceso violento y complejo de apropiación de recursos naturales y de degradación ambiental, pero que puede ser justificado por la renta que genera y la oportunidad que tiene el Estado para invertir dichas ganancias en el desarrollo del país. Empero, la concentración de la economía del país en este tipo de actividades generan varios problemas ya que dichas economías son vulnerables al depender de los precios internacionales de comercialización del producto. Por el contrario, este tipo de extractivismo puede ser catalogado como agresivo, ya que se enmarca el neo-extractivismo denominado por Gudynas en el que se observa un papel activo del Estado para captar parte de la riqueza que generan los sectores extractivos (Gudynas, 2009, 208).

\section{POLÍTICA EXTRACTIVA PETROLERA EN ECUADOR, UNA POLÍTICA DE AMPLIACIÓN DE LA CAPACIDAD PRODUCTIVA Y DE RESERVAS}

En el contexto de la caracterización del extractivismo petrolero en el Ecuador, la historia ha demostrado que la política pública ha sido una de ampliación de reservas y la producción. Esto ha sido constante, especialmente a partir del denominado boom petrolero de la década de los setenta.

El resultado de la implantación de esta política pública fueron miles de barriles de petróleo producidos, pero también varias comunidades 
afectadas, en especial pueblos indígenas, ya que la localización geográfica de dichas actividades extractivas ha sido la Amazonía del Ecuador. Esto, sin duda, comprueba la violencia y la violación a derechos humanos con las que han sido emprendidas las actividades extractivas.

Entre los hitos importantes de la implantación de esta política petrolera en Ecuador tenemos:

- La firma del Protocolo de Río de Janeiro, que dividió el territorio de muchos pueblos indígenas, pero que tuvo como trasfondo un conflicto entre dos petroleras (Almeida y Proaño, 2008, 45).

- El descubrimiento de grandes reservas de petróleo en el nororiente del país (zona de Lago Agrio) por el consorcio Texaco - Gulf en el año de 1967 (Acosta, 2000, 49)

- El inició un proceso de colonización para ofrecer mano de obra a la industria petrolera (Almeida y Proaño, 2008, 46) y de apropiación de territorios en la Amazonía a través de la Ley de Reforma Agraria y Colonización de 1967, y la Ley especial para adjudicación de tierras baldías a favor de la colonización espontánea del Oriente ecuatoriano.

- El contacto y cambio cultural forzado (Franco, 2006, 278) con las poblaciones que vivían en las cercanías del río Curaray, y que tuvo la ayuda del Instituto Lingüístico de Verano (ILV) (Almeida y Proaño, 2008, 27).

- En la década de los setenta, se observó un desplazamiento forzado de los pueblos indígenas Waorani (Acosta, Aguilar, Quevedo, Spurrier, y Marchán, 1986, 29). Incluso se habló de la desaparición del pueblo conocido como Tetetes (Franco, 2006, 278) debido a la explotación petrolera (OILWATCH, 2001, 20).

- En la década de los ochenta existió gran actividad referente a perforaciones de desarrollo y la incorporación de nuevos campos a la producción (Banco Central del Ecuador, 1990, 15). Además, se iniciaron rondas internacionales de licitaciones para concesionar bloques a empresas privadas transnacionales (Acción Ecológica, 2011, 4); y se dio la salida de la compañía Texaco de la Amazonía que dio lugar al inicio de un largo proceso judicial para reclamar por los daños ambientales (Gordillo, 2003, 239). 
- En la década de los noventa se crearon las condiciones para transferir los bienes públicos al capital privado (Acción Ecológica, 2011, 4).

- En la década de los años 2000 destaca la defensa territorial emprendida por el Pueblo Kichwa de Sarayaku frente a la arremetida de la empresa CGC y el apoyo del Estado ecuatoriano para emprender actividades extractivas dentro de su territorio, lo cual tuvo como resultado que el caso sea llevado al Sistema Interamericano de Derechos Humanos en el año 2012.

Esto ha sido en ejemplo de cómo en todos estos años se observa que la explotación petrolera siempre ha ido en perjuicio de los pueblos indígenas en la medida en que los campos petroleros y su producción han ido en aumento. En este caso, la política extractiva petrolera en el Ecuador siempre se ha dado en función de proyectos que buscan la ampliación de la producción y las reservas hidrocarburíferas.

El período entre 2008 y 2016 no fue la excepción, y se puede visualizar en el Plan Nacional del Buen Vivir de los períodos 2009-2013 y 2013-2017 que delimitan la política extractiva petrolera actual del país.

En los planes correspondientes se establece la necesidad del cambio de modelo, es decir el paso de un esquema primario exportador y extractivista a uno que privilegie la producción diversificada y ecoeficiente (SENPLADES, 2009, 329-330) y a una economía del conocimiento (SENPLADES, PNBV, 2013, 82). Esta ha sido la justificación para plantear el incremento de reservas y producción petrolera como fuente de riqueza social indispensable en el tránsito hacia el nuevo modelo (SENPLADES, 2009, 336-340).

Esta política se encuentra reproducida también en el Plan Estratégico Ministerial 2014-2017 elaborado por el Ministerio de Recursos Naturales No Renovables, hoy Ministerio de Hidrocarburos, que señala el incremento de la contribución al desarrollo nacional del sector hidrocarburífero como las actividades a ser emprendidas por el Ministerio.

Lo mismo ocurre en el artículo 15 del Reglamento de Aplicación de la Ley Reformatoria a la Ley de Hidrocarburos de 2010, que establece como principios de Política Hidrocarburífera: 


\section{Gráfico 2: Principios de la Política Hidrocarburífera}

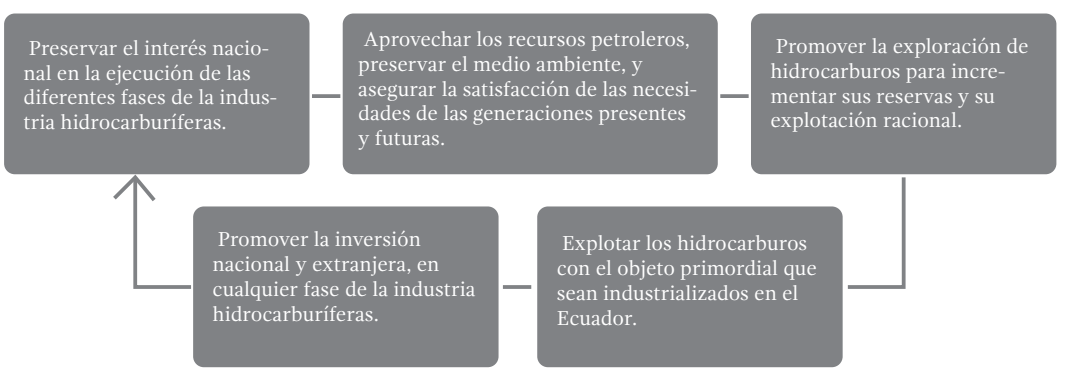

Fuente: Reglamento de la Ley Reformatoria a la Ley de Hidrocarburos de 2010 Elaboración: Propia

Los proyectos en los que se expresa esta política extractiva son la Ronda Sur Oriente y la explotación de los bloques 31 y 43 (o ITT).

Sobre la Ronda Sur Oriente, se trata de un proyecto planteado desde el año 2000, pero que tuvo la resistencia territorial de varios pueblos indígenas como la FIPSE (hoy NASHE), la FICSH, la FINAE (hoy NAE) y el Pueblo Kichwa de Sarayaku (Melo, 2012, 108). Pese a estos procesos de resistencia, se tienen como hechos relevantes:

- En el 2010 el Estado firmó un convenio con la compañía Coreana de Petróleos para proyectar las oportunidades de exploración y explotación de petróleo en la Amazonía suroriente (Melo, 2012, 104);

- En octubre de 2011 se anunció un nuevo mapa petrolero por parte del Secretario de Hidrocarburos (Melo, 2012, 105);

- Entre los meses de mayo y octubre de 2012 se inició un proceso de consulta a las comunidades indígenas (Mazabanda, 2013, 4);

- El 28 de noviembre de 2012 con la convocatoria a las rondas de licitación de 13 bloques mediante la modalidad de contratación por prestación de servicios (Secretaría de Hidrocarburos, 2016), a los que se suman 3 bloques que estarían bajo la operación de la empresa estatal Petroamazonas, y 5 bloques que serán licitados a futuro, según se mencionó en el transcurso del 2013 (Mazabanda, 2013, 4). 
Según la información de la Secretaría de Hidrocarburos, para el 28 de noviembre de 2013 se anunciaron cuatro ofertas para los bloques $29,28,79$ y 83 , lo que conllevó a que en abril de 2015 se firme contrato para actividades extractivas en el bloque 28 (Acción Ecológica, 2015) y para enero de 2016 la firma de los contratos para la exploración de los bloques 79 y 83 (Araujo, 2016).

Por otro lado, el proyecto de explotación dentro de los bloques 31 y 43 significó también una ampliación en la frontera de explotación petrolera en el país. Este proyecto tuvo como antecedente una iniciativa de dejar bajo tierra el crudo del bloque ITT que inició en el año 2007. Sin embargo, el 23 de agosto de 2013 el Presidente de la República dio por terminada dicha iniciativa y solicitó a la Asamblea Nacional que declare de interés nacional la explotación petrolera de los bloques 31 y 43 dentro del Parque Nacional Yasuní en un área no superior al uno por mil.

Al respecto, hay que mencionar que en el informe realizado por el Ministerio de Justicia para fundamentar su solicitud a la Asamblea sobre el proyecto ITT, se cambió el mapa de presencia histórica de los pueblos Tagaeri-Taromenane que habitan en esa zona a uno que muestra su distribución. Esto le permitió al Estado no incurrir en la prohibición de realizar actividades extractivas dentro de territorios pueblos indígenas aislados del artículo 57 de la Constitución, pese a que han existido informes y pronunciamientos en los que se ha puesto en evidencia su presencia en dichos bloques petroleros (Colectivo Geografía Crítica del Ecuador, 2017).

Pese a esto, el 4 de octubre de 2013 la Asamblea Nacional resolvió declarar de interés nacional la explotación petrolera de los bloques 31 y 43 dentro del Parque Nacional Yasuní en una extensión no mayor al uno por mil. El 6 de enero de 2014, se asignó el Bloque 43 a Petroamazonas EP, mientras que años antes, en el 2009, la misma empresa asumió el Bloque 31. Actualmente, estos dos bloques cuentan con licenciamiento ambiental para la realización de actividades de explotación petrolera.

En razón de lo expuesto, se puede visibilizar que la política extractiva petrolera en Ecuador siempre fue la de ampliar su frontera, encontrándose como constante hechos de violencia y violación a derechos humanos, en especial con los pueblos indígenas como ya se mencionó. 


\section{UNA POLÍTICA PÚBLICA EN EL ESTADO CONSTITUCIO- NAL DE DERECHOS Y JUSTICIA}

Frente a esta realidad, en la que incluso la Constitución legitima este tipo de actividades, cabe una alternativa que pueda dar un tratamiento distinto a estas actividades.

La misma Constitución de 2008 planteó un nuevo modelo de Estado en el que el ejercicio de los derechos y la consecución de la justicia en todas las esferas de la sociedad pasaron a tomar un rol protagónico. Por esta razón, desarrolla una serie de principios que orientan las actividades de las autoridades y limitan el ámbito de sus funciones para el cumplimiento de los derechos y la generación de condiciones sociales más equitativas y justas.

En este marco estatal que plantea la Constitución de 2008 se inscribe el Buen Vivir o Sumak Kawsay, entendido como un proceso mediante el cual los individuos conviven de forma armónica entre ellos y la naturaleza (Larrea, 2015, 2), y como un valor de la vida que exalta la riqueza espiritual de los pueblos y de la naturaleza, y el espacio como seres que conviven con los humanos (Kowii, 2014).

Cobra sentido entonces el reconocimiento y ejercicio de los derechos humanos y los derechos de la naturaleza, puesto que los seres humanos necesitamos de la naturaleza para vivir y esta a su vez necesita también de los seres humanos (Ávila, 2011, 47).

Los derechos humanos han tenido un desarrollo importante tanto en la legislación como en la jurisprudencia nacional e internacional. Esta ha sido una herramienta importante para las personas que han luchado y han defendido sus territorios y sus formas de vida frente a la imposición de actividades extractivas.

Sin embargo, un avance importante de la Constitución se refiere al reconocimiento de derechos a la naturaleza. Dentro de este contexto, los artículos 71 y 72 reconocen el derecho al respeto integral de su existencia, al mantenimiento y regeneración de sus ciclos vitales, y a la restauración integral de los ecosistemas. Este ha sido considerado un reconocimiento, conceptual y filosófico, vinculado con la visión de un país plural para impulsar el Buen Vivir y la Plurinacionalidad, y que significa una ruptura con paradigmas jurídicos, éticos y hasta religiosos (Melo, 2010, 6), entre ellos la mercantilización de la natura- 
leza. Producto de este reconocimiento innovador de derechos a la naturaleza, se establece la necesidad de adoptar medidas de prevención y de restricción de actividades, lo cual se relaciona con el artículo 14 de la Constitución que reconoce el derecho de la población a vivir en un medio sano y ecológicamente equilibrado.

Es en este marco constitucional en el que las políticas públicas deben actuar como una manifestación del ejercicio del poder estatal. En efecto, el artículo 85.1 de la Constitución determina que las políticas públicas deben orientarse a hacer efectivos el buen vivir y todos los derechos, y se formularán a partir del principio de solidaridad.

En el caso en que una política pública vulnere o amenace con vulnerar derechos, esta deberá reformularse o se deberán adoptar medidas alternativas que concilien los derechos en conflicto según el artículo 85.2 de la Constitución.

Por lo tanto, frente a la implementación de una política que vulnere derechos, como es la extractiva petrolera, es obligación del Estado reformularla o cambiarla por una que respete los derechos reconocidos en la Constitución.

\section{CONCLUSIÓN}

Como hemos visto, la política extractiva petrolera tiene como sustento una legislación, una institucionalidad, y una forma de gestión que ha sido realizada por el Estado. Su implementación se la ha venido justificando como mecanismo indispensable para el desarrollo económico del país al vincularse con la realización de políticas sociales.

Sin embargo, el énfasis que ha puesto el Estado en este tipo de actividades ha demostrado lo contrario, puesto que se tiene una economía dependiente y vulnerable tanto frente a la bonanza por los altos precios de los recursos naturales en el mercado mundial como cuando existe una baja en los precios del mismo. Además, las rentas obtenidas de estos recursos no necesariamente han sido utilizadas para la implementación de otras políticas sociales.

Esta realidad también ha venido acompañada de procesos violentos de apropiación territorial, sobretodo de los territorios de los pueblos indígenas; de daños ambientales, en especial debido al tipo de acti- 
vidad en sí. En este punto, resulta problemático afirmar un balance positivo del extractivismo petrolero en el Ecuador.

Por lo tanto, al ser una política pública que ha vulnerado derechos humanos y derechos de la naturaleza a través de los años debe reformularse, o en el mejor de los casos, deben plantearse medidas alternativas dentro del contexto de una época post petrolera para el país.

Por ejemplo, Eduardo Gudynas plantea ya un proceso de transiciones de un extractivismo agresivo, como el relatado en este texto, a uno indispensable donde permanecen las actividades necesarias, según condiciones sociales y ambientales, y vinculadas a cadenas productivas nacionales y regionales, para nutrir redes de consumo verdaderamente enfocadas en la calidad de vida (Gudynas, Caminos para las transiciones postextractivistas, 2012, 176).

Esto es una tarea larga y compleja puesto que requiere el trabajo tanto de la sociedad como del Estado. El papel del Derecho en este tema también debe replantearse, puesto que es posible avistar todavía tensiones en la Constitución respecto a una visión mercantilista de los recursos naturales que permite su explotación frente a la otra, que maneja una búsqueda del Buen Vivir en el cual seres humanos y naturaleza vivan en armonía a través del goce efectivo de sus derechos. En este sentido, debe cambiar las dinámicas en cuanto a la estructura económica del país y al relacionamiento del Estado con las comunidades donde existan proyectos extractivos y la sociedad en general.

\section{REFERENCIAS BIBLIOGRÁFICAS}

Acción Ecológica. (2011). Ampliación de la frontera petrolera: Traición al Sumak Kaysay. Quito: Acción Ecológica.

Acción Ecológica. (2015). Explotación Petrolera en el bloque 28 afectaría cuencas hídricas del centro sur de la Amazonía. Obtenido de http://www.accionecologica.org/editoriales/1799-explotacion-petrolera-en-el-bloque-28-afectaria-cuencas-hidricas-del-centro-sur-de-la-amazonia 
Acosta, A. (2000). Ecuador post petrolero. Quito: Acción Ecológica.

Acosta, A. (2006). Breve Historia Económica del Ecuador. Quito: Corporación Editora Nacional.

Acosta, A. (2009). La maldición de la abundancia. Quito: Abya Yala.

Acosta, A. (2009). La maldición de la abundancia. Quito: Ediciones Abya-Yala.

Acosta, A. (2015). Después del saqueo: Caminos hacia el posextractivismo. En Fundación Heinrich Böll, Más allá de la sobreexplotación - Alternativas latinoamericanas al extractivismo (p. 12-17). America Latina: Fundación Heinrich Böll.

Acosta, A. (2015). Poscrecimiento y Posextractivismo: Dos caras de la misma transformación cultural. Obtenido de La Línea de Fuego: http://lalineadefuego.info/2015/03/31/poscrecimiento-y-posextractivismo-dos-caras-de-la-misma-transformacion-cultural-por-alberto-acosta/

Acosta, A., Aguilar, M., Quevedo, C., Spurrier, W. y Marchán, C. (1986). Ecuador: petróleo y crisis económica. Quito: Instituto Latinoamericano de Investigaciones Sociales, ILDIS.

Aleman, A. (2001). Community, Higher Education and the Challenge of Multiculturalism. Teachers College Record.

Almeida, A. y Proaño, J. (2008). Tigre, Águila y Waorani. Una sola selva, una sola lucha. Quito: Acción Ecológica.

Araujo, A. (2016). Dos contratos petroleros se firmarán la próxima semana. El Comercio. Obtenido de: http://www.elcomercio.com/actualidad/contratos-petroleros-firmaran-ecuador-hidrocarburos.html.

Ávila, R. (2011). El derecho de la naturaleza: fundamentos. En C. Espinosa Gallegos-Anda, \& C. Pérez Fernández, Los Derechos de la Naturaleza y la Naturaleza de sus Derechos (p. 35-74). Quito: Ministerio de Justicia, Derechos Humanos y Cultos.

Ávila, R. (2011). Neoconstitucionalismo transformador. Quito: Abya Yala. 
Ávila, R. (2012). Aproximaciones: nuevas construcciones de indicadores. En F. Cevallos, Educación y buen vivir. Reflexiones sobre su construcción (p. 153-155). Quito: Contrato Social por la educación en el Ecuador.

Azamar, A. y Ponce, J. I. (2014). Extractivismo y desarrollo: Los recursos minerales en México. Revista Problemas del Desarrollo, 137-158.

Bahá'í International Community. (1995). The Prosperity of Mankind. Haifa: Baha'i International Community.

Banco Central del Ecuador. (1990). La actividad petrolera en el Ecuador en la Década de los 80. Quito: Subgerencia de Publicaciones Técnicas. Gerencia Técnica del Banco Central del Ecuador.

Chemerinsky, E. (2015). Constitutional Law principles and policies. New York: Wolters Kluwer.

Coatsworth, J. H. (2004). Globalization, Growth, and Welfare in History. En Suárez-Orozco, M. M. y Qin-Hilliard, D. B. Globalization Culture and Education in the New Millennium. London: University of California Press, Ltd.

Colectivo Geografía Crítica del Ecuador. (2017). Colectivo Geografía Crítica del Ecuador. Obtenido de https://geografiacriticaecuador.org/2014/04/09/manifiesto-de-la-geografia-por-el-yasuni-el1x1000-no-existe-en-el-territorio/

Corte Constitucional del Ecuador, Caso 0004-10-SIN-CC, 0004-10-SINCC (Corte Constitucional del Ecuador 29 de Abril de 2010).

Corte Constitucional del Ecuador, Caso 001-09-SEE-CC, 001-09-SEECC (Corte Constitucional del Ecuador 20 de Febrero de 2009).

Corte Constitucional del Ecuador, Caso 001-12-SIC-CC, 001-12-SICCC (Corte Constitucional del Ecuador 25 de Enero de 2012).

Corte Constitucional del Ecuador, Caso 015-10-SEE-CC, 015-10-SEECC (Corte Constitucional del Ecuador 8 de Julio de 2010). 
Dávalos, P. (2013). Las falacias del discurso extractivista. Obtenido de América Latina en movimiento online: http://www.alainet.org/ images/Las\%20falacias\%20del\%20extractivismo_PD.pdf

Dávalos, P. (2014). Alianza PAIS o la reinvención del poder. Siete ensayos sobre el posneoliberalismo en el Ecuador. Bogotá: Ediciones desde abajo.

Dye, T. (2012). Undestanding Public Policy. New Jersey: Prentice Hall.

El Universo. (2015). Finanzas anuncia nuevo recorte en presupuesto de Ecuador del 2015: \$ 800 millones.

Eslava, J. y Puente, C. (s.f.). Análisis de las políticas públicas: una aproximación desde la teoría de los sistemas sociales. CENDEX.

Facultad de Economía de la PUCE. (2015). Reporte de Consistencia Macroeconómica. Quito: PUCE.

Franco, J. C. (2006). Responsabilidad cultural: el reto de la inclusión de los derechos culturales. En G. Fontaine, Petróleo y Desarrollo Sostenible en Ecuador (275-186). Quito: FLACSO Ecuador, ILDIS-FES, Petrobras Ecuador.

Fundación Heinrich Böll. (2015). Más allá de la sobreexplotación - Alternativas latinoamericanas al extractivismo. América Latina: Fundación Heinrich Böll.

Fundación Mil Hojas. (2015). Así Ecuador dejó de ser un país petrolero. Obtenido de Plan V: http://www.planv.com.ec/investigacion/investigacion/asi-ecuador-dejo-ser-un-pais-petrolero/pagina/0/1

Gonzáles, A. (2008). El tema de los indicadores en las políticas públicas de derechos humanos -propuestas para su conceptualización y abordajeObtenido de Repositorio UASB: http://hdl.handle.net/10644/1000

Gordillo, R. (2003). ¿El Oro del Diablo? Ecuador: historia del petróleo. Quito: Corporación Editora Nacional.

Gudynas, E. (2009). Diez tesis urgentes sobre el nuevo extractivismo. Contextos y demandas bajo el progresismo sudamericano actual. En Varios Autores, Extractivismo, política y sociedad (187-225). Quito: Centro Andino de Acción Popular y Centro Latino Americano de Ecología Social. 
Gudynas, E. (2012). Caminos para las transiciones postextractivistas. En Alayza, A. y Gudynas, E. Transiciones: Postextractivismo y alternativas al extractivismo en el Perú (165-192). Lima: Red Peruana por una Globalización con Equidad - RedGE / Centro Peruano de Estudios Sociales - CEPES .

Gudynas, E. (2013). Extracciones, extractivismos y extrahecciones. Un marco conceptual sobre la apropiación de recursos naturales. $\mathrm{Ob}$ servatorio del Desarrollo, 1-18.

Harvey, D. (2005). El "nuevo" imperialismo: acumulación por desposesión. Socialist register, 99-129.

Heibert, P. G. (1983). Cultural Anthropology. Cite: Baker Book House.

Hernández, I., Rodríguez, M. C. y Moreno, Á. (2004). Mercado vs. Estado: un debate inconcluso. Economía y Desarrollo, 39-52.

Hofstede, J. G. and Pedersen, P. B. (2002). Exploring Culture. Maine: Intercultural Press Inc.

Huntington, S. P. (2003). The Clash of Civilizations and the Remarking of World Order. New York: Simon \& Schuster Paperbacks.

Játiva, J. (2010). Plan de desarrollo del campo Tumali--Petroamazonas. Quito: Escuela Politécnica Nacional.

Kelman, S. (1992). La Política Pública en el Estado Moderno. (C. Piña, Trad.) Buenos Aires: Grupo Editor Latinoamericano.

Kohls, L. R. (1996). Survival Kit for Overseas Living: For Americans Planning to Live and Work Abroad. Yarmouth: Intercultural Press.

Kowii, A. (2014). Sumak Kawsay, la palabra usurpada. Plan V. 5p.

Larrea, C. (2015). El Buen Vivir frente a las nociones convencionales de desarrollo. Quito.

Lenzerini, F. and Borelli, S. (2012). Cultural Heritage, Cultural Rights, Cultural Diversity: New Developments in International Law. Leiden: Brill. 
Lobos, D. A. (2013). Los territorios de la desposesión: los enclaves y la logística como territorialización del modelo extractivo sudamericano. Revista NERA, 43-54.

May, C. I. (2013). Constitutional Law National Power and Federalism. New York: Wolters Kluwer.

Mazabanda, C. (2013). Consulta Previa en la Décimo Primera Ronda Petrolera - ¿Participación masiva de la ciudadanía? Quito: Fundación Pachamama.

Melo, M. (2010). Derechos de la Pachamama: un paradigma emergente frente a la crisis ambiental global. Aportes Andinos No. 27, 7p.

Melo, M. (2012). La nueva ronda petrolera y el derrumbe del paradigma constitucional. Horizonte de los derechos humanos, Ecuador 2012, 103-117.

Ministerio de Hidrocarburos. (2015). La inversión en el sector petrolero garantiza sostenibilidad de ingresos para el Estado. Obtenido de http://www.hidrocarburos.gob.ec/la-inversion-en-el-sector-petrolero-garantiza-sostenibilidad-de-ingresos-para-el-estado/

Molina, E. (2014). Los efectos jurídicos de la reforma a la Ley de Hidrocarburos, publicada en el Registro Oficial No. 244, en los contratos de prestación de servicios entre el Estado ecuatoriano y las operadoras de exploración y explotación de hidrocarburos. Quito: PUCE.

Mukherjee, J. (2014). Conflict Resolution in Multicultural Societies : The Indian Experience. New Delhi: SAGE Publications India Pvt Ltd.

Naciones Unidas. (2012). Indicadores de Derechos Humanos. Guía para la medición y la aplicación. Nueva York \& Ginebra: Naciones Unidas.

Nederveen Pieterse, J. (2007). Ethnicities and Global Multiculture: Pants for an Octopus. Lanham: Rowman \& Littlefield Publishers, Inc.

Nordgren, R. (2002). Globalization and Education: What Students Will Need To Know And Be Able To Do In The Global Village. The Phi Delta Kappan, 318-321.

Oilwatch. (2001). La manera occidental de extraer petróleo. Quito: Oilwatch. 
Oxfam Internacional. (2009). Contra la 'Maldición de los Recursos' Naturales. Cómo pueden y deben beneficiarse las personas pobres de la renta de las extractivas. Obtenido de Informe de Oxfam: http://www. extractivismo.com/documentos/OxfamContraMaldicionRentaExtractivas.pdf

Petit Primera, J. G. (2013). La teoría económica del desarrollo desde Keynes hasta el nuevo modelo neoclásico del crecimiento económico. Revista Venezolana de Análisis de Coyuntura, 123-142.

Pollock, D. C. and Van Reke, R. E. (1999). The Third Culture Kid Experience-Growing Up Among Worlds. Maine: Intercultural Press, Inc.

Rapto, 665 - 06 (Corte Nacional de Justicia Primera Sala de lo Penal 30 de Octubre de 2006).

Redacción Plan V. (2015). Las diez posibles salidas a la crisis económica según Ortiz. Obtenido de http://www.planv.com.ec/historias/politica/ diez-posibles-salidas-la-crisis-economica-segun-ortiz

Ruiz Vieytez, E. J. (2014). United in Diversity? : On Cultural Diversity, Democracy and Human Rights. Bruxelles: P.I.E-Peter Lang S.A., Éditions Scientifiques Internationales.

Santos Ragus, E. J. (2007). Perceptions of Language Educators About Global Education as part of the Content-Based Learning in Teaching English as a Foreign Language. Obtenido de Tesis de Maestría Universidad San Francisco de Quito: http://repositorio.usfq.edu.ec/handle/23000/260

Scott, E. P. (2007). The Difference - How the Power of Diversity Creates Better Groups, Firms, Schools, and Societies. New Jersey: Princeton University Press.

Secretaría de Hidrocarburos. (2016). Ronda Sur Oriente. Obtenido de http://www.rondasuroriente.gob.ec/

SENPLADES. (2009). PNBV. Plan Nacional para el Buen Vivir 20092013. Quito: SENPLADES.

SENPLADES. (2011). Guía para la Formulación de Políticas Públicas Sectoriales. Quito: Suplemento del Registro Oficial Nº 184. 
SENPLADES. (2013). Empresas Públicas y Planificación. Su rol en la transformación social y productiva. Quito: SENPLADES.

SENPLADES. (2013). PNBV. Plan Nacional para el Buen Vivir 20132017. Quito: SENPLADES.

Serrano Narváez, H. (2013). Caso Chevron-Texaco: cuando los pueblos toman la palabra. Quito: Universidad Andina Simón Bolívar.

Skelton, M., Wigford, A., Harper, P. and Reeves, G. (2002). Beyond Food, Festivals, and Flags. Educational Leadership Journal, 60.

Svampa, M. (2013). «Consenso de los Commodities» y lenguajes de valoración en América Latina. Nueva Sociedad No 244, 30-46.

Tied, P. L. and Tied, I. M. (2002). Multicultural Teaching. Boston: PEC.

Wright, R. (2010). The Moral Animal. New York: Pantheon Books.

Recibido: $12 / 02 / 2017$

Aprobado para publicación: 27/05/2017

Felipe Castro León (LL.B, LL.M): Abogado por la Pontificia Universidad Católica del Ecuador. Responsable del Observatorio de Justicia Constitucional de la Universidad Andina Simón Bolívar-Ecuador y de la Pontificia Universidad Católica de Ecuador.

Correo electrónico: fcastroleon@gmail.com 